\title{
Experimental entanglement of six photons in graph states
}

\author{
CHAO-YANG LU1*, XIA0-QI ZHOU1 ${ }^{1}$, OTFRIED GÜHNE², WEI-BO GA0 ${ }^{1}$, JIN ZHANG ${ }^{1}$, ZHEN-SHENG YUAN ${ }^{1}$, \\ ALEXANDER GOEBEL ${ }^{3}$, TAO YANG ${ }^{1}$ AND JIAN-WEI PAN ${ }^{1,3 *}$
}

\author{
${ }^{1}$ Hefei National Laboratory for Physical Sciences at Microscale and Department of Modern Physics, University of Science and Technology of China, Hefei, Anhui 230026, \\ People's Republic of China \\ ${ }^{2}$ Institut für Quantenoptik und Quanteninformation, Österreichische Akademie der Wissenschaften, Technikerstraße 21A, A-6020 Innsbruck, Austria \\ ${ }^{3}$ Physikalisches Institut, Universität Heidelberg, Philosophenweg 12, D-69120 Heidelberg, Germany \\ *e-mail: cylu@mail.ustc.edu.cn; jian-wei.pan@physi.uni-heidelberg.de
}

Published online: 14 January 2007; doi:10.1038/nphys507

Graph states ${ }^{1-3}$-multipartite entangled states that can be represented by mathematical graphs-are important resources for quantum computation ${ }^{4}$, quantum error correction ${ }^{3}$, studies of multiparticle entanglement ${ }^{1}$ and fundamental tests of non-locality ${ }^{5-7}$ and decoherence ${ }^{8}$. Here, we demonstrate the experimental entanglement of six photons and engineering of multiqubit graph states $^{9-11}$. We have created two important examples of graph states, a six-photon Greenberger-HorneZeilinger state ${ }^{5}$, the largest photonic Schrödinger cat so far, and a six-photon cluster state ${ }^{2}$, a state-of-the-art 'one-way quantum computer'4. With small modifications, our method allows us, in principle, to create various further graph states, and therefore could open the way to experimental tests of, for example, quantum algorithms $\mathrm{s}^{4,12}$ or loss- and fault-tolerant oneway quantum computation ${ }^{13,14}$.

Entanglement lies at the heart of quantum mechanics and plays a crucial role in quantum-information processing. Many efforts have been undertaken to create, in particular, multipartite entangled states in different physical systems ${ }^{15-18}$, where maximally up to eight ions have been entangled ${ }^{18}$. In recent years, special types of multipartite entangled states, the graph states $^{1-3}$, have become the centre of attention. They can be associated with graphs where each vertex represents a qubit prepared in the state $(1 / \sqrt{2})(|0\rangle+|1\rangle)$ and each edge represents a controlled phase gate having been applied between the two connected qubits. An interesting feature is that many entanglement properties of graph states are closely related to their underlying graphs ${ }^{1}$. Besides their thought-provoking theoretical structure, the graph states have also provided new insights into studies of nonlocality ${ }^{6,7,19,20}$ and decoherence ${ }^{8}$ and served as essential resources for various quantum-information tasks ${ }^{3,4}$, most prominently as the exceptionally universal resource for one-way quantum computation $^{4}$. Encouraging progress ${ }^{9-14,16}$ has been achieved in this direction, especially in the linear optics regime. Yet a major challenge ahead lies in the experimental generation of multiqubit graph states.

Of special interest in the graph-state family are the Greenberger-Horne-Zeilinger (GHZ) states and the cluster states. Experimentally, six-atom GHZ states ${ }^{17}$ and four-photon cluster states $^{16}$ have been realized. Here, we report the creation of six-photon GHZ states and cluster states with verifiable six-partite entanglement. To do so, we start from three Einstein-PodolskyRosen (EPR) entangled photon pairs in the state

$$
\left|\Phi^{+}\right\rangle_{i j}=\frac{1}{\sqrt{2}}\left(|H\rangle_{i}|H\rangle_{j}+|V\rangle_{i}|V\rangle_{j}\right),
$$

where $H$ and $V$ denote horizontal and vertical polarization, and $i$ and $j$ label the spatial modes of the photons (see Fig. 1a). We superpose photons in mode 2 and 3 (4 and 5) at polarizing beam splitters (PBSs). As the PBS transmits $H$ and reflects $V$ polarization, only if both incoming photons have the same polarization can they go to different outputs ${ }^{21,22}$. Thus, a coincidence detection of all six outputs corresponds to the state

$$
\begin{aligned}
\left|G_{6}\right\rangle= & \frac{1}{\sqrt{2}}\left(|H\rangle_{1}|H\rangle_{2}|H\rangle_{3}|H\rangle_{4}|H\rangle_{5}|H\rangle_{6}\right. \\
& \left.+|V\rangle_{1}|V\rangle_{2}|V\rangle_{3}|V\rangle_{4}|V\rangle_{5}|V\rangle_{6}\right),
\end{aligned}
$$

which is a six-photon GHZ state, exhibiting an equal superposition of two maximally different quantum states.

By applying a Hadamard gate on photon 4 before it enters into the PBS (see Fig. 1a), the above scheme can be readily modified to generate a six-photon cluster state. It can be considered in two steps: (1) combine photons 2 and 3, such that, on the basis of a coincidence detection, we get a four-photon GHZ state $(1 / \sqrt{2})\left(|H\rangle_{1}|H\rangle_{2}|H\rangle_{3}|+\rangle_{4}+|V\rangle_{1}|V\rangle_{2}|V\rangle_{3}|-\rangle_{4}\right)$, where $| \pm\rangle=(1 / \sqrt{2})(|H\rangle \pm|V\rangle)$; (2) combine photons 4 and 5 , and by a similar reasoning we obtain what we call here a six-photon cluster state

$$
\begin{aligned}
\left|C_{6}\right\rangle= & \frac{1}{2}\left(|H\rangle_{1}|H\rangle_{2}|H\rangle_{3}|H\rangle_{4}|H\rangle_{5}|H\rangle_{6}\right. \\
& +|H\rangle_{1}|H\rangle_{2}|H\rangle_{3}|V\rangle_{4}|V\rangle_{5}|V\rangle_{6} \\
& +|V\rangle_{1}|V\rangle_{2}|V\rangle_{3}|H\rangle_{4}|H\rangle_{5}|H\rangle_{6} \\
& \left.-|V\rangle_{1}|V\rangle_{2}|V\rangle_{3}|V\rangle_{4}|V\rangle_{5}|V\rangle_{6}\right) .
\end{aligned}
$$


a

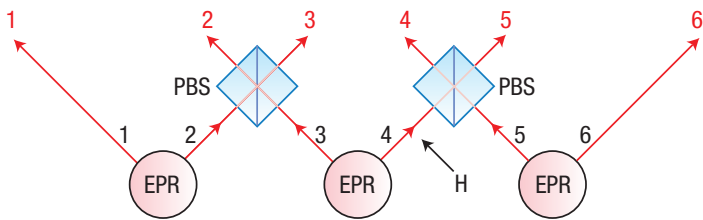

b<smiles>COc1ccc(O)cc1</smiles><smiles>COC(=O)O</smiles>

G

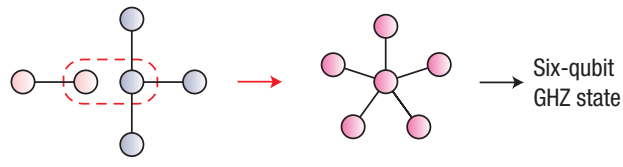

d

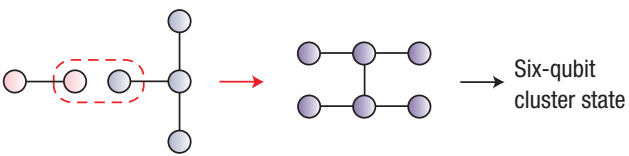

Figure 1 Scheme to generate the six-photon graph states and their representations in the graph-state picture. a, The six-photon GHZ state (1) and cluster state (2) are created by combining three pairs of entangled photons at PBSs. The Hadamard gate $(\mathrm{H})$ is inserted for generation of the cluster state. $\mathbf{b}-\mathbf{d}$, Underlying graph of the six-photon graph states and how they are created by post-selected fusion operations. The graph state can be thought of as being constructed by first preparing the qubits at each vertex in the state $|+\rangle=(1 / \sqrt{2})(|H\rangle+|V\rangle)$ and then applying controlled phase gates between pairs of neighbouring qubits. Here, we use the post-selected fusion operations, that is, combining photons at PBS, to generate multiqubit graph states efficiently. In the star graph, we refer to the central node as the root and the others as leaves.

For an intuitive understanding, in Fig. 1 we show the underlying graph of the above states and how they grow from smaller (twoqubit) graph states. Up to local unitary transformations, the GHZ states correspond to star-shaped graphs, and the cluster states to lattice graphs. The effect of combining two photons at PBS can be described by the operator $|H H\rangle\langle H H|+| V V\rangle\langle V V|$, leading to the fusion of two separate graph states into a single one $\mathrm{e}^{10,11}$. Specifically, Fig. 1c (d) shows that when a two-qubit graph state is combined with the root (leaf) node of a four-qubit star graph, a six-qubit GHZ (cluster) state is produced.

A nice feature of the graph-state representation is that many properties of the graph states and their potential use in quantuminformation processing can be revealed by their underlying graph. For example, the star-graph states have multiple leaf nodes, which are referred to as microclusters in refs 9,13 and can be used in the so-called parallel fusion for building up large cluster states. The graph of the six-qubit cluster state (2) forms a standard quantum circuit under the one-way computer model ${ }^{4}$. Moreover, its geometry embodies a tree-shaped graph, which is the basic building block for loss-tolerant one-way quantum computing ${ }^{14}$. Another interesting feature of the cluster state is that not only itself but even the remaining mixed four-qubit state after two qubits have been traced out, leads to a GHZ argument for non-locality ${ }^{6}$, showing a surprisingly strong entanglement persistency.

Let us now proceed with the experimental demonstration. In our experiment, we use spontaneous parametric down-conversion to produce entangled photons ${ }^{23}$. We made various efforts to prepare high-brightness and stable sources of entangled photons (see the Methods section). The set-up is illustrated in Fig. 2. A pulsed ultraviolet laser successively passes through three $\beta$-barium borate (BBO) crystals to generate entangled photon pairs in spatial modes $1-2,3-4$ and $5-6$. The photon pairs are prepared in the state $\left|\Phi^{+}\right\rangle$ with an average two-fold coincidence count of about $9.3 \times 10^{4} \mathrm{~s}^{-1}$ and a visibility of $93 \%(91 \%)$ in the $H / V(+/-)$ basis. We then superpose photons 2 (4) and 3 (5) at the PBS. To achieve good spatial and temporal overlap, the photons are spectrally filtered $\left(\Delta \lambda_{\text {FWHW }}=3.2 \mathrm{~nm}\right)$ and detected by fibre-coupled single-photon detectors. By making fine adjustments of delay $\Delta d_{1}\left(\Delta d_{2}\right)$, we are able to observe interference fringes of four-photon entanglement with a visibility of $73 \%(71 \%)$ in mode $1-2-3-4$ (3-4-5-6), indicating that the post-selected fusion operations have been successfully implemented (see the Supplementary Information).
Now we analyse the experimental data of six-photon graph states and characterize the entanglement produced here. Let us first discuss to what extent the desired states were produced and the presence of genuine multipartite entanglement. The quality of the states can be judged by the fidelity, that is, the overlap of the produced state with the desired one. The notion of genuine multipartite entanglement characterizes whether generation of the state requires interaction of all parties: a pure state $|\Psi\rangle$ is called biseparable, whenever a grouping of the six parties into two groups $G_{A}$ and $G_{B}$ can be found, such that the state is a product state, that is $|\Psi\rangle=|\alpha\rangle_{G_{A}} \otimes|\beta\rangle_{G_{B}}$, otherwise it is a genuine multipartite entangled state. Consequently, a mixed state is called biseparable, if it is a mixture of biseparable pure states, otherwise it is a genuine multipartite entangled state.

To prove multipartite entanglement, we use the method of entanglement witnesses ${ }^{25}$. An entanglement witness is an observable that has a positive expectation value on all biseparable states. Thus, a negative expectation value proves the presence of genuine multipartite entanglement. In what follows, we derive efficient entanglement witnesses that are both robust against realistic noise and economical for experimental efforts.

For the six-photon GHZ state (1), we use the witness ${ }^{25}$

$$
W_{\mathrm{G}}=\frac{I}{2}-\left|G_{6}\right\rangle\left\langle G_{6}\right|
$$

where $I$ denotes the identity operator. We decompose $\left|G_{6}\right\rangle\left\langle G_{6}\right|$ into locally measurable observables

$$
\left|G_{6}\right\rangle\left\langle G_{6}\right|=\frac{1}{2}\left[(|H\rangle\langle H|)^{\otimes 6}+(|V\rangle\langle V|)^{\otimes 6}\right]+\frac{1}{12} \sum_{n=-2}^{3}(-1)^{n} M_{(n)}^{\otimes 6},
$$

where $M_{(n)}=\cos (n \pi / 6) \sigma_{x}+\sin (n \pi / 6) \sigma_{y}$ are measurements in the $x-y$ plane. To implement this witness, seven measurement settings are required. Figure 3 shows the measurement results, yielding $\operatorname{Tr}\left(W_{\mathrm{G}} \rho_{\exp }\right)=-0.093 \pm 0.025$, which is negative by 3.7 standard deviations and thus proves the presence of genuine sixpartite entanglement.

From the expectation value of the witness, we can directly determine the obtained fidelity as $F_{G_{6}}=\left\langle G_{6}\left|\rho_{\exp }\right| G_{6}\right\rangle=$ $0.593 \pm 0.025$, where $\rho_{\exp }$ denotes the experimentally produced 


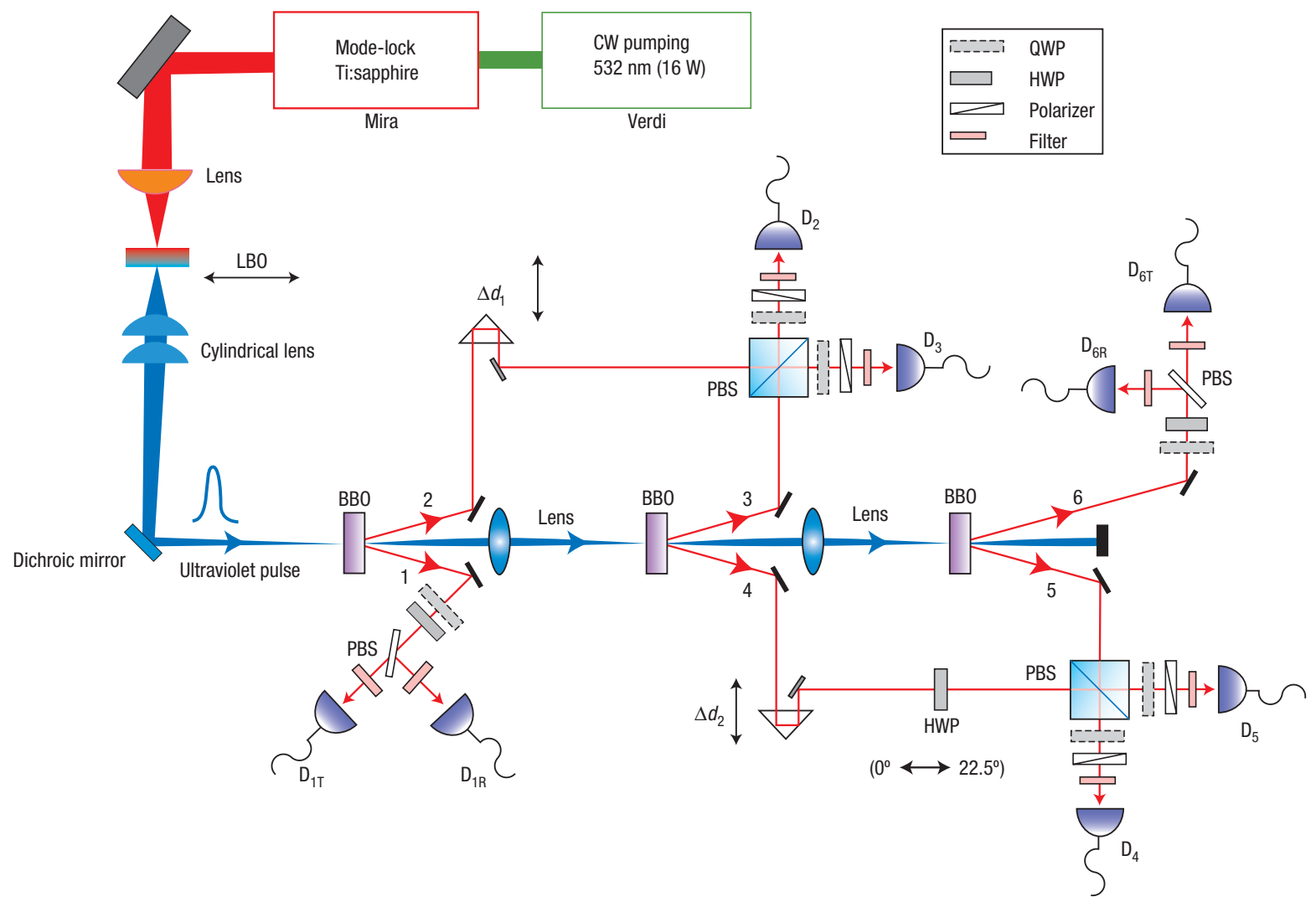

Figure 2 Experimental set-up for the generation of six-photon graph states. Pumped by a continuous-wave (CW) green laser, the mode-locked Ti:sapphire laser outputs a pulsed infrared laser with a central wavelength of $788 \mathrm{~nm}$, a pulse duration of $120 \mathrm{fs}$ and a repetition rate of $76 \mathrm{MHz}$, which passes through an LBO crystal (mounted on a motorized translation stage) and is up-converted to ultraviolet $(\lambda=394 \mathrm{~nm}$ ). The ultraviolet laser beam is circularized and focused on the three BBO crystals to produce three pairs of entangled photons. The entangled photons are spectrally filtered by narrow-band filters (with a peak transmission rate of $\sim 98 \%$ ) and then detected by fibre-coupled single-photon detectors $\left(D_{1 T}, \ldots, D_{6 R}\right)$. We use a programmable multichannel coincidence unit to register the multi-fold coincidence events. For polarization analysis, halfand quarter-wave plates (HWP, QWP) together with polarizers or PBSs are used. By changing the angle $(\theta)$ of the HWP at path 4, our set-up is tunable to generate the six-photon GHZ states $\left(\theta=0^{\circ}\right)$ and cluster state $\left(\theta=22.5^{\circ}\right)$.

state. This is a considerable improvement over the fidelity of the six-atom GHZ states ${ }^{17}(F=0.509 \pm 0.004)$.

For the cluster state (2), a possible witness would be $W_{\mathrm{C}}=I / 2-\left|C_{6}\right\rangle\left\langle C_{6}\right|$ (ref. 26). Similar to the constructions of ref. 26 , we use a slightly different witness $\widetilde{W}_{\mathrm{C}}$, the implementation of which requires only six measurements (see the Methods section). Figure 4 shows the measurement results in basis $\sigma_{z}^{\otimes 3} \sigma_{x}^{\otimes 3}\left(\sigma_{x}^{\otimes 3} \sigma_{z}^{\otimes 3}\right)$, which together with those of the four other bases $\sigma_{z}^{\otimes 3} M_{( \pm 1)}^{\otimes 3}$ and $M_{( \pm 1)}^{\otimes 3} \sigma_{z}^{\otimes 3}$ (see the Supplementary Information), gives $\operatorname{Tr}\left(\widetilde{W}_{\mathrm{C}} \rho_{\exp }\right)=-0.095 \pm 0.036$. Thus, the genuine six-partite entanglement of the cluster state is also proved. Furthermore, from this result, we can obtain a lower bound of the fidelity of our cluster state as $F_{\mathrm{C}_{6}} \geq 0.595 \pm 0.036$.

For an investigation of the bipartite entanglement properties of these graph states, we estimate the entanglement of formation from the expectation value of the witness ${ }^{27}$. Here, different bipartitions arise when the six parties are divided into two groups. The entanglement of formation $E_{\mathrm{F}}(\rho)$ is an entanglement measure for bipartite systems, quantifying how many EPR pairs are needed for the formation of the state ${ }^{28}$. For the GHZ state (1), we find that for all bipartitions at least $E_{\mathrm{F}}\left(\rho_{\exp }\right) \geq 0.073 \pm 0.032$. For the cluster state $(2), E_{\mathrm{F}}\left(\rho_{\exp }\right)$ is also always positive, for some bipartitions it is even $E_{\mathrm{F}}\left(\rho_{\exp }\right) \geq 0.729 \pm 0.106$. A full discussion, also for a different entanglement measure, is given in the Supplementary Information.
The imperfections of our graph states are mainly caused by two reasons. First, high-order emissions of entangled photons give rise to the undesired components in the $H / V$ basis (see Fig. 3a). Second, the partial distinguishability of independent photons causes some incoherent mixtures. In spite of the imperfections, genuine entanglements of the six-photon graph states are strictly confirmed. It is possible to improve the fidelity in future experiments, for example, by using photon-number discriminating detectors to filter out the events of double emissions of photon pairs. Moreover, graph states with high purity can be obtained efficiently using the existing entanglement purification scheme ${ }^{29}$. The linear optical elements such as the PBS may offer a highaccuracy tool for this $\operatorname{task}^{30}$. It leaves a crucial open question of how to reach the noise thresholds for optical cluster-state quantum computation ${ }^{13}$.

Some further remarks are warranted here. We generate the graph states conditioned on there being one and only one photon in each of the six outputs. This post-selective feature, on the one hand, together with the fusion method, provides a flexible and economical way to create various multiphoton graph states. Slight modifications of our experimental set-up will readily allow the creation of many other graph states, for example, six-qubit linear and Y-shaped graph states (see the Supplementary Information). Such a fascinating capacity creates 

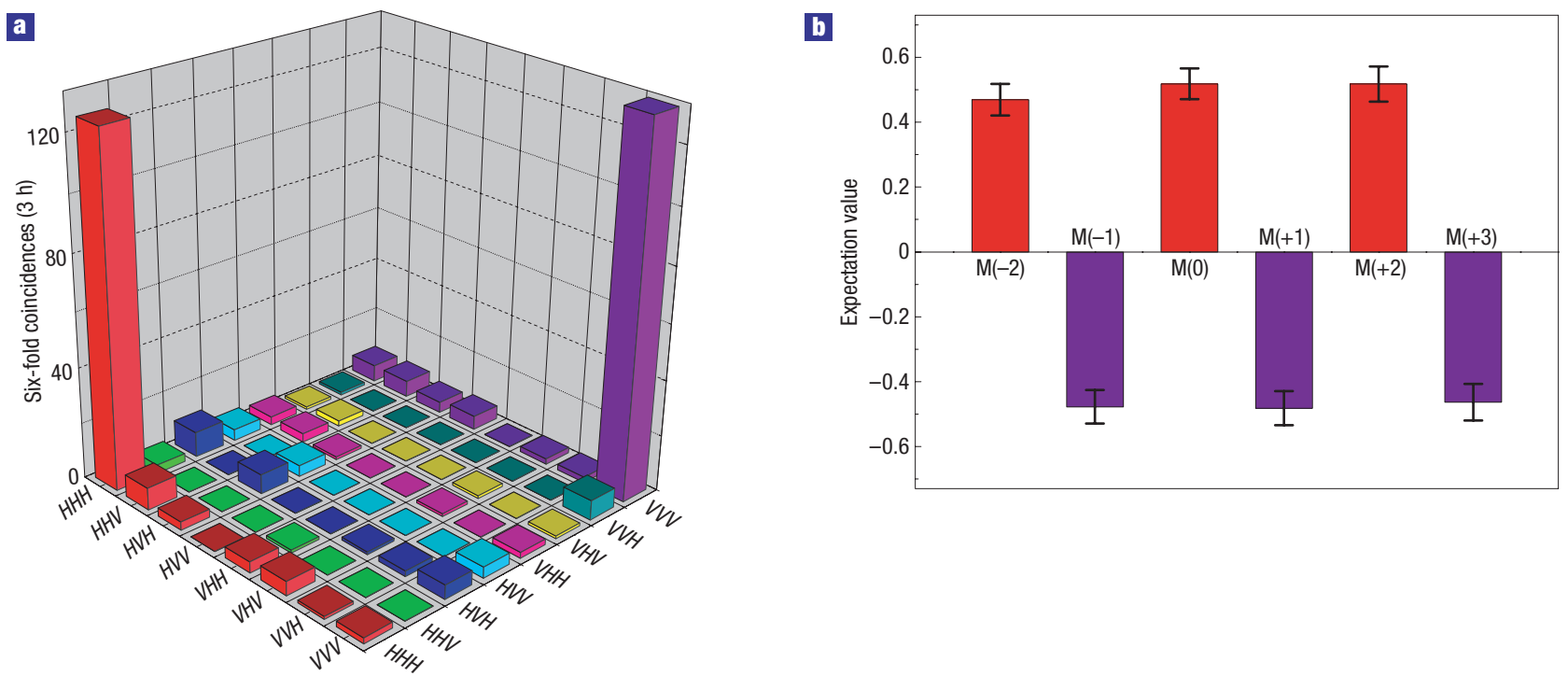

Figure 3 Experimental result of the six-photon GHZ state (1). a, Six-fold coincidence counts in $H / V$ basis in $3 \mathrm{~h}$. $\mathbf{b}$, The expectation values of $M_{(n)}^{\otimes 6}$, each derived from a complete set of 64 six-fold coincidence events in $2 \mathrm{~h}$ in measurement basis $|H\rangle \pm \mathrm{e}^{i n \pi / 6}|V\rangle$. The error bars represent one standard deviation, deduced from propagated poissonian counting statistics of the raw detection events.
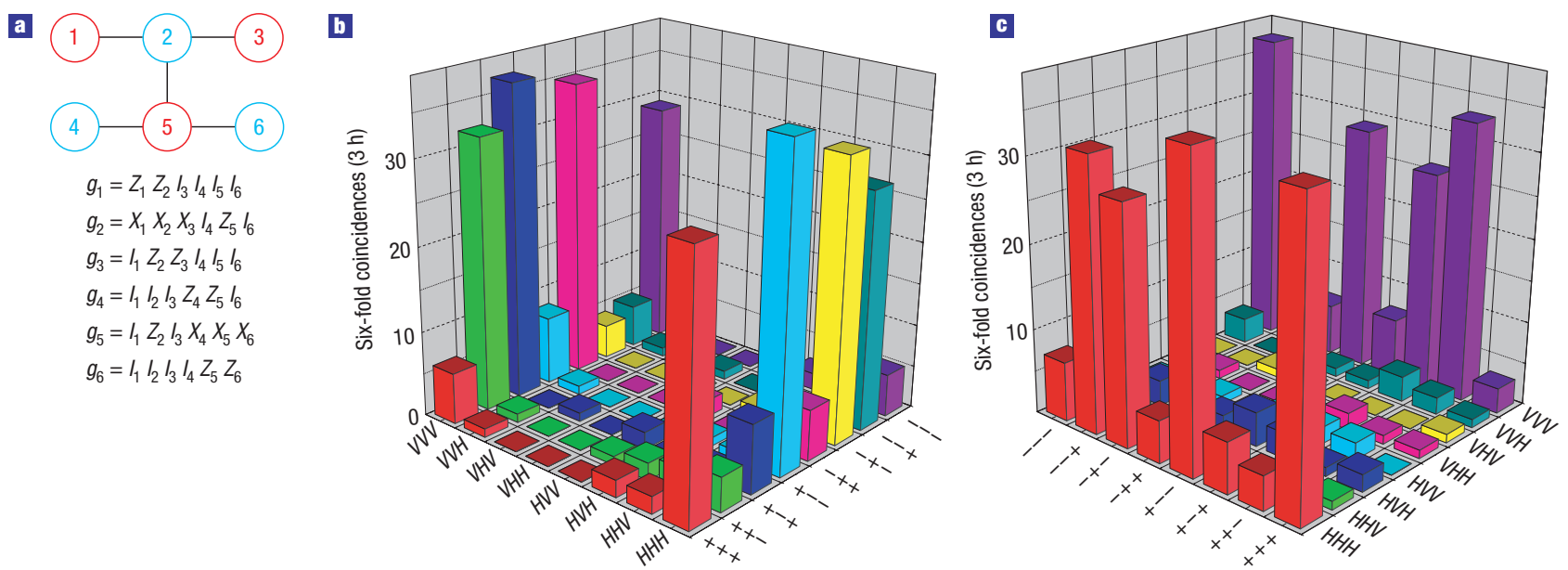

Figure 4 The stabilizer operators and experimental result of the six-photon cluster state (2). a, The graph corresponds to the cluster state $\left|C_{6}\right\rangle$ under $\mathrm{H}$ transformations on qubits $1,3,4$ and 6 and its stabilizer operators $g_{i}$, where $i$ labels the qubits and $X=\sigma_{x}, Y=\sigma_{y}, Z=\sigma_{z}$. The graph state is a common eigenstate of these stabilizer operators, that is, $g_{i}\left|C_{6}\right\rangle=\left|C_{6}\right\rangle$, which describe the correlations in the state. The cluster state is the unique state fulfilling this, which allows for an alternative definition of it. b,c, Six-fold coincidence counts measured in the $\sigma_{z}^{\otimes 3} \sigma_{x}^{\otimes 3}$ and $\sigma_{x}^{\otimes 3} \sigma_{z}^{\otimes 3}$ basis in $3 \mathrm{~h}$, each signals the observation of an eigenstate of the stabilizer operator $\left(g_{i}\right)$ with the corresponding eigenvalue of $v_{j}=+1$ or -1 . From the probabilities of multiphoton detections $p_{j}, j=1,2, \ldots, 64$, we can then compute the expectation values of stabilizer operators by $\operatorname{Tr}\left(g_{i} \rho\right)=\sum_{j=1}^{64} p_{j} v_{j}$.

a useful multiqubit graph-state test-bed. On the other hand, this feature does not prohibit subsequent applications such as tests of quantum non-locality ${ }^{5-7}$ and in-principle verifications of linear optical quantum-information processing tasks where photons need to be eventually detected. Finally, concerning the scalability issue, we refer to ref. 11, which has shown that if combined with quantum memory, the post-selection method can even be used for scalable generation of tree-graph states using realistic linear optics. Along this line, however, technically extensive efforts still need to be undertaken to make a quantum memory usable for this purpose.
In summary, we have realized two special graph states, the six-photon GHZ state, the largest photonic Schrödinger cat so far, and the six-photon cluster state-a state-of-the-art oneway quantum computer. We have demonstrated the ability to entangle six photons and to engineer multiqubit graph states, and have created a versatile test-bed for experimental investigations of one-way quantum computation ${ }^{4}$, quantum error correction ${ }^{3}$, studies of multiparticle entanglement ${ }^{1}$ and foundational tests of quantum physics ${ }^{5-8}$. Combined with quantum memory, our experimental method could lead to the generation of large-scale tree-graph states ${ }^{11}$. The high efficiency and flexibility of the six- 
photon graph-state generation we demonstrated here suggest that photons manipulated with linear optics are promising candidates for engineering of multiqubit graph states. Various applications of our six-photon graph-state test-bed can be imagined. For instance, the six-qubit cluster states allow full implementations of the quantum game of prisoners' dilemma ${ }^{12}$ and a proof-ofprinciple demonstration of the basic elements of loss-tolerant one-way quantum computation ${ }^{14}$. Most remarkably, the six-qubit star-ring graph state corresponds to the codeword and encoding procedure of the five-qubit quantum error-correction code that is able to correct all one-qubit errors ${ }^{3}$. In addition, our six-photon cluster state also enables a novel test of non-locality, namely a GHZ argument of non-locality for mixed states ${ }^{6}$. Lastly, the graph-state test-bed is well suited for studies of the stabilities of different types of multiparticle entanglement (for example, GHZ and cluster) under the influence of decoherence, which may provide experimental evidence for the surprising conclusion in ref. 8 that genuine entanglement of a macroscopic number of particles is possible and can persist for timescales that are independent of the size of the system.

\section{METHODS}

\section{OPTIMIZING THE SIX-PHOTON SET-UP}

To get high-brightness entangled photons, similarly to ref. 24 we used the high-power Verdi-18/Mira-900F femtosecond laser system that outputs a stable pulsed infrared laser with a power of $2.4 \mathrm{~W}$. In addition, we adopted a new three-BBO-crystal configuration for a more flexible optimization of three independent photon pairs. Moreover, the use of narrow-band filters in our experiment with peak transmission rates as high as $98 \%$ considerably improved the collection efficiency. To make our set-up stable, we first made efforts to minimize the fluctuation of the pump power. As shown in Fig. 2, we mounted the $\mathrm{LiB}_{3} \mathrm{O}_{5}(\mathrm{LBO})$ crystal on a motorized translation stage controlled by a computer program to avoid damage to the crystal by the focusing laser beam. By doing this, we achieved both good up-conversion efficiency $(\sim 38 \%)$ and long life time for LBO, making it a very stable ultraviolet laser source with a power of $0.91 \mathrm{~W}$ that typically drops by less than $1.5 \%$ over weeks. Our feedback control system has greatly improved the stability of the down-conversion rates: the measured coincidence counts typically fluctuate by $\sim 3 \%$ over two weeks, whereas without it the counts drop significantly after about $15 \mathrm{~min}$ (see the Supplementary Information for a curve of the measured two-fold coincidences over time). Second, we used a programmable multi-channel coincidence unit that allowed us to simultaneously register any possible coincidence detection between the inputs. Aside from the desired six-fold coincidence events, we also registered other combinations (such as single counts and two-fold coincidences), so we were able to track the performance of our system in real time. Any power drift could be deduced from the recorded count rates, and finally the six-fold coincidences were corrected for their difference. Thus, we were able to achieve a stable set-up, good collection efficiencies and three EPR pairs with high visibilities.

\section{CLUSTER-STATE WITNESS CONSTRUCTION}

The witness for the cluster state (2) can be constructed as follows. Using the results of ref. 26, the observable $W_{\mathrm{C}}=I / 2-\left|C_{6}\right\rangle\left\langle C_{6}\right|$ is a witness detecting genuine multipartite entanglement around the cluster state. Then, we consider the observable

$$
\begin{aligned}
\widetilde{W}_{\mathrm{C}}= & \frac{3}{2} I-\prod_{i=1,3,5} \frac{g_{i}+I}{2}-\prod_{i=2,4,6} \frac{g_{i}+I}{2} \\
& -\frac{1}{2}\left(I \otimes A_{0}+A_{0} \otimes I\right)-\left(A_{1} \otimes B_{1}+B_{1} \otimes A_{1}\right) \\
= & \frac{I}{2}-\left|C_{6}\right\rangle\left\langle C_{6}|+| \widetilde{C}_{6}\right\rangle\left\langle\widetilde{C}_{6}\right|,
\end{aligned}
$$

where $g_{i}$ denotes the stabilizing operators of the cluster state (see Fig. 4a).
Furthermore, we use $A_{0}=I-|H H H\rangle\langle H H H|-| V V V\rangle\langle V V V|$, $A_{1}=|V V V\rangle\langle V V V|-| H H H\rangle\langle H H H|$ and $B_{1}=\left(M_{(1)}^{\otimes 3}+M_{(-1)}^{\otimes 3}\right) / 2 \sqrt{3}$, where $M_{(i)}$ is defined as for the GHZ state (see equation (3)). Finally, $\left|\widetilde{C}_{6}\right\rangle$ denotes a cluster state with different signs, namely $\left|\widetilde{C}_{6}\right\rangle=(-|H H H H H H\rangle+$ $|H H H V V V\rangle+|V V V H H H\rangle+|V V V V V V\rangle) / 2$.

It is clear that $\widetilde{W}_{\mathrm{C}}-W_{\mathrm{C}} \geq 0$, which implies that $\widetilde{W}_{\mathrm{C}}$ is a valid witness ${ }^{26}$. Furthermore, this implies that the fidelity of the cluster state can be estimated as $F_{\mathrm{C}_{6}}=\left\langle C_{6}\left|\rho_{\exp }\right| C_{6}\right\rangle \geq(1 / 2)-\left\langle\widetilde{W}_{\mathrm{C}}\right\rangle$.

The witness $\widetilde{W}_{\mathrm{C}}(4)$ detects genuine entanglement from the states of the form $\rho(p)=p\left|C_{6}\right\rangle\left\langle C_{6}\right|+(1-p) I / 64$ for $p>0.5$. The determination of the expectation value of the witness $\widetilde{W}_{\mathrm{C}}$ requires six measurement settings, namely $\sigma_{z}^{\otimes 3} \sigma_{x}^{\otimes 3}, \sigma_{x}^{\otimes 3} \sigma_{z}^{\otimes 3}, \sigma_{z}^{\otimes 3} M_{( \pm 1)}^{\otimes 3}$ and $M_{( \pm 1)}^{\otimes 3} \sigma_{z}^{\otimes 3}$. The results are shown in Fig. 4 in the main text and in Supplementary Information, Fig. S3.

Received 18 September 2006; accepted 5 December 2006; published 14 January 2007.

References

1. Hein, M., Eisert, J. \& Briegel, H.-J. Multiparty entanglement in graph states. Phys. Rev. A 69 062311 (2004)

2. Briegel, H. J. \& Raussendorf, R. Persistent entanglement in arrays of interacting particles. Phys. Rev. Lett. 86, 910-913 (2001).

3. Schlingemann, D. \& Werner, R. F. Quantum error-correcting codes associated with graphs. Phys. Rev. A 65, 012308 (2002).

4. Raussendorf, R. \& Briegel, H. J. A one-way quantum computer. Phys. Rev. Lett. 86, 5188-5191 (2001). 5. Greenberger, D. M., Horne, M. A., Shimony, A. \& Zeilinger, A. Bell's theorem without inequalities. Am. J. Phys. 58, 1131-1143 (1990).

6. Scarani, V. et al. Nonlocality of cluster states of qubits. Phys. Rev. A 71, 042325 (2005).

7. Gühne, O. et al. Bell inequalities for graph states. Phys. Rev. Lett. 95, 120405 (2005).

8. Dür, W. \& Briegel, H.-J. Stability of macroscopic entanglement under decoherence. Phys. Rev. Lett. 92, 180403 (2004)

9. Nielsen, M. A. Optical quantum computation using cluster states. Phys. Rev. Lett. 93, 040503 (2004).

10. Browne, D. E. \& Rudolph, T. Resource-efficient linear optical quantum computation. Phys. Rev. Lett. 95, 010501 (2005).

11. Bodiya, T. P. \& Duan, L.-M. Scalable generation of graph-state entanglement through realistic linear optics. Phys. Rev. Lett. 97, 143601 (2006).

12. Paternostro, M. et al. Hybrid cluster state proposal for a quantum game. New J. Phys. 7, 226 (2005).

13. Dawson, C. M., Haselgrove, H. L. \& Nielsen, M. A. Noise thresholds for optical quantum computers. Phys. Rev. Lett. 96, 020501 (2006).

14. Varnava, M., Browne, D. E. \& Rudolph, T. Loss tolerance in one-way quantum computation via counterfactual error correction. Phys. Rev. Lett. 97, 120501 (2006).

15. Zhao, Z. et al. Experimental demonstration of five-photon entanglement and open-destination teleportation. Nature 430, 54-58 (2004).

16. Walther, P. et al. Experimental one-way quantum computing. Nature 434, 169-176 (2005).

17. Leibfried, D. et al. Creation of a six-atom 'Schrödinger cat' state. Nature 438, 639-642 (2005).

18. Häffner, H. et al. Scalable multiparticle entanglement of trapped ions. Nature 438, 643-646 (2005).

19. Walther, P., Aspelmeyer, M., Resch, K. J. \& Zeilinger, A. Experimental violation of a cluster state Bell inequality. Phys. Rev. Lett. 95, 020403 (2005).

20. Kiesel, N. et al. Experimental analysis of a four-qubit photon cluster state. Phys. Rev. Lett. 95, 210502 (2005).

21. Zeilinger, A., Horne, M. A., Weinfurter, H. \& Zukowksi, M. Three-particle entanglements from two entangled pairs. Phys. Rev. Lett. 78, 3031-3034 (1997).

22. Pan, J.-W. et al. Experimental demonstration of four-photon entanglement and high-fidelity teleportation. Phys. Rev. Lett. 86, 4435-4438 (2001).

23. Kwiat, P. G. et al. New high-intensity source of polarization-entangled photon pairs. Phys. Rev. Lett. $75,4337-4341$ (1995)

24. Zhang, Q. et al. Experimental quantum teleportation of a two-qubit composite system. Nature Phys. 2, 678-682 (2006)

25. Bourennane, M. et al. Experimental detection of multipartite entanglement using witness operators Phys. Rev. Lett. 92, 087902 (2004).

26. Toth, G. \& Gühne, O. Entanglement detection in the stabilizer formalism. Phys. Rev. A 72 022340 (2005).

27. Gühne, O., Reimpell, M. \& Werner, R. F. Estimating entanglement measures in experiments. Preprint at $<$ http://arxiv.org/abs/quant-ph/0607163> (2006)

28. Bennett, C. H. et al. Mixed-state entanglement and quantum error correction. Phys. Rev. A 54, 3824-3851 (1996).

29. Dür, W., Aschauer, H. \& Briegel, H.-J. Multiparticle entanglement purification for graph states. Phys. Rev. Lett. 91, 107903 (2003).

30. Pan, J.-W. et al. Experimental entanglement purification of arbitrary unknown states. Nature $\mathbf{4 2 3}$ $417-422(2003)$.

\section{Acknowledgements}

We thank H. J. Briegel, D. Browne, L.-M. Duan, T. Rudolph and S. Yu for helpful discussions. This work was supported by the National Natural Science Foundation of China, the Chinese Academy of Sciences. This work was also supported by the Alexander von Humboldt Foundation, the Marie Curie Excellence Grant of the EU, the FWF, the DFG and EU (Scala, Olaqui, Prosecco, QICS, Quprodis). Correspondence and requests for materials should be addressed to C.-Y.L. or J.-W.P. Supplementary Information accompanies this paper on www.nature.com/naturephysics.

Competing financial interests

The authors declare that they have no competing financial interests.

Reprints and permission information is available online at http://npg.nature.com/reprintsandpermissions/ 